\title{
ALIMENTAÇÃO E FECUNDIDADE DE PLANORBIDEOS CRIADOS EM LABORATÓRIO: IV - HELISOMA DURYI (WETHERBY, 1879). (PULMONATA, PLANORBIDAE) *
}

\author{
Roberto Milward-de-Andrade** \\ Sandra Maria Maruch**** \\ Maria Juana Custa ***
}

MII. WARD-DE-ANDraDe. R. et al. Alimentaçio ' fecandidade de planorbideos criados em laboratorios: IV - Helisuma duryi (Wetherby, 1879). (Pulmonata. Planorbidae). Rev. Saúde públ., S. Panlo, 12:90-8, 1978.

RESEno: Utilizando dois tipos distintos de alimentos: "Aquariol" (alimento utilizado para alimentar pexes ornamentais) e alface, isolados a associados. na criaça de Helisoma (Seminolina) duryi (Wetherby, 1879) em laboratório. foi verificado que: 1 . Os caramujos alimentados com alface fresca liberaram. em 17 semanas, 826 desovas oll 17.707 ovos. 2. Os especimens alimentados com "aquariol" depuseram 531 desovas ou 11.361 ovos. 3. O oferecimento dos dols materiais associados resultou no aumento da fertilidade, obtendo-se, assim. 1.355 desolas ou 34.645 olos. 4. Em termos médios, cada conjunto de aninze exemplares (com 10 a $14 \mathrm{~mm}$ de diametro) liberon, diariamente, $95,5-148,8$ e 291,1 olos/dia, segundo o tipo de alimento consumido: "aquariol", alface fresca, ou ambos associados. O nimero de desovas/dia, na mesma ordem enunciada. foi de $4.5-6.9$ e 11,4 respectivamente. 5. Os exemplares utilizados descendiam de uma 'epa supostamente recém introduzida no municipio de Formosa, $G O$ (Brasil) é originária da Região Neártica (EUA). Foi comentada a possibilidade de controle biológico de diferentes espécies de Biomphalaria atraves de $\mathrm{H}$. duryi - questio sobre a qual lềm desenvolvendo investigaşos biologicas e cológicas.

Unrermos: Planorbideos. Helisuma duryi.

\section{NT RODLCAO}

A familia Planorbidat engloba 6 gêneros: Helisoma Swainson, 1840: Drepanotrema Fischer \& Crosse, 1880; Plesiophisa Fis. cher, 1883; Biomphalaria Preston, 1910: Acrorbis Odhener, 1937: Antillorbis Har- ly \& Hubendick, $196+$ e 19 espécies identificadas no Brasil, ate agora.

O géneto Helisoma, cuja distribuiçàn geográfica primitiva está associacla à Regiãu Neártica (América do Norte), foi, recente-

* Traballo conplementudo com auxilio do CXPa. Apresentado à $29 a$ Reunia Anual da Sociedade Basileira palia o Progresso da Cifncia isBPC). São Paulo, 6-13/julho:197i.

** Do Centro de Pesquisas "Renf́ Firhou" dit Fundação Oswaldo Cruz - Caixa Postal 17t:" - 30000 - Belo Horlzonte. MG - Brasil e di Lniversidade Federal de Minas Gerais Belo Horizonte. MIG - Brasil 
MILWARD-DE-ANDRADE, $R$. et al. Alimentuğa e fecundidide de platnorbídeos criados em laboratório: IV - Helisoma duryi (Wetherby, 1879). (Pulnonati, Planorhidze). Rer. Saúde puibl.. S. Paulo, 12:90-8, 1978.

mente, introduzido no Brasil por acuaristas (Paraense: 1975).

A espécie $H$. (Seminola) duryi foi capturada no Estado de Goiás, numa lagoa "in sympatry with Biomphalaria straminea, B. schrammi, Drepanotrema anatinum, D. lucidum and Plesiophysa ornata, besides ancylids, physids and ampullariids" (Paraenses 1976).

Ao que parece, trata-se de espécie pouco conhecida do ponto de vista biológico e ecológico.

Recentemente, no Egito, Abdallah e Nasr $^{1}$ (1973) observaram, experimentalmente no laboratório e no campo, yue $H$. duryi pode obstar o desenvolvimento de populações de Biomphalaria alexandrina e de Bulinus trucatus, em decorrência da açãu de substância inibidora sobre ovos e formas jovens das espécies nomeadas. Assinalaram, ainda, que Helisoma duryi seria util competidor de hospedeiros intermediários de Schistosoma.

Apresentaremos daclos decorrentes da uriação, em laboratório, de Helisoma (S.) duryi descendentes de exemplares capturados na Lagoa da Pedra, distrito de Santa Rosa, municipio de Formosa, Estado de Goiás e gentilmente enviados ao Laboratório de Ecologia do Centro de Pesquisas "René Rachou" da Fundação Oswaldo Cruz (CPqRR/FIOCRUZ) pelo Dr. W. Lobat" Paraense, em 1975.

Os experimentos foram realizados segundo técnica anteriormente estabelecida para estudo de alimentação de Biomphalaria tenagophila, B. straminea e $B$. glabrata (Milward-de-Andrade e Carvalho $\%$, 1972: Milward-de-Andrade e col. s,6. 1973, 1974).

\section{MATERIAL E METODOS}

Recipientes - Durante as 17 semanas consecutivas de observações, nove conjuntos de cinco exemplares de helisoma foram mantidos en 9 pequenas bacias plásticas, opacas ,de cor azul, com largura de $20 \mathrm{~cm}$, altura de $10 \mathrm{~cm}$ e comprimento de $30 \mathrm{~cm}$.
Áglla - A yuantidade de água utilizada toi sempre igual a 3 litros, sendo renovada a intervalos de 20 dias, ncasiões em que as bacias eram convenientemente lavadas. As amostras da água provinham de fonte natural, isenta de poluição humana, localizada no bairro da Serra, Belo Horizonte, $.11 G$.

Temperatura $\left({ }^{\circ} \mathrm{C}\right)$ - Diariamente, entre y $~ 12$ horas, registrava-se as temperaturas da água e do ambiente. Os daclos apresentados (Tabela 1) referem-se aos valores médios semanais, calculados a partir das anotaçóes diárias, procediclas de dezembro 1976 a março/1977.

Planorbideos utilizados - Em cada uma das nove bucias plásticas utilizadas, foram colocados cinco exemplares pigmentados de H. (S.) dutryi, cujo diâmetro das conchas variava de 10 a $14 \mathrm{~mm}$. Estes exemplares foram criados no laboratório, em aquário de vidro, com água da rede de abastecimento da cidade, senclo rotineiramente alimentados com folhas de alface fresca.

Ao longo dos 119 dias ou 17 semanas de ubservações diárias, ocorrell a morte de 19 espécimens, que foram substituidos por "utros de iguais diâmetros e da mesma procedência, isto é, do aquário mencionado no item precedente.

Alimentação oferccida - A alimentação oferecida aos caramujos estava representada, de um lacio, por abundantes fragnentos, frescos, de Lactuca sativa L. (Compositae) $e$, de outro, por quantidades previamente determinadas, isto é, $3,0 \mathrm{mg} / \mathrm{dia}$ ) de "Aquariol" - produto industrializado para alimentaçāo) de peixes ornamentais, composto, segundo o fabricante, de farinhas de carne, de peixe e de camarão, mescladas à farinha de milho (fubá), parcialmente desidratada.

Aos helisomas contidos no primeiro grupo cie três bacias (1, 2 e 3 ) foram proporcionados apenas alface fresca, cujos abundantes fragmentos eram diariamente 
MILWARD-DE-ANDRADE. R. et al. Almuntação e fecundirade de planorbidens criados em laboratório: If - Helisoma duryi (Wothrohy 1879). (Pumonata. Planotbidael. Rer. Satide públ., S. Paulo. 12:94-5. 1978.

substituidos por novas porçoes de material fresco.

Os espécimens colocados no seguncin grupo de bacias ( 4,5 e 6 ) furam diariamente alimentados com ambos us produfus. associados: altace - ayuariol.

Finalmente, os caramujos distribuidos ro terceiro grupo de bacias $(7,8$ e 9 ) tiveram como raçāo diária $3.0 \mathrm{~m}$ @g de ạ̣uariul.

\section{RESULTADOS}

Temperatura $\left({ }^{\circ} \mathrm{C}\right)$ - Durante o transcurso dos 119 dias do experimento, whservou-se que as temperaturas minimas, absilutas, para a água e para o ambiente. foran, respectivamente, de $23^{\circ}$ e $23,5^{\circ} \mathrm{C}$.

O menor valor médio, semanal, de temperatura da água foi igual a $23,5^{\circ} \mathrm{C}$ ( ta. e 10a. semanas); a média semanal mais elevada alcançon $27.7^{\circ} \mathrm{C}$ (16a. semana).

Também em termo médio, semanal. a temperatura do ar mais baixa foi de $23,0^{\circ} \mathrm{C}$ (9a. semana), encuanto a mais elevada atingiu us $28,2^{\circ} \mathrm{C}$ ( $16 \mathrm{a}$. semana) (Tabela 1 ).

As temperaturas máximas, absolutas. para a água e para o ar não ultrapassaram: a $28^{\circ}$ e $28,5^{\circ} \mathrm{C}$. respectivamente, ao long das 17 semanas de ubservaçoes (dezembro 76 a março/77).

Ovos e desoras, segundo o tipo de allmento - Considerando os dois tipos de materiais oferecidos aos caramujos, ohservou-se os seguintes resultados, quand, isolados ull associados.

1. Alface - Houve sensivel variaçāı numérica de desovas semanalmente liberadas; por exemplo, de apenas o na primeira semana para 103 na décima primeira -- tuta-
T A B E L 1

Temperaturas (oC) médias, do ar e da água, durante o transcurso de 17 semanas em que foram criados quinze exemplares de Helisoma duryi, com dois tipos diferentes de alimentos. Belo Horizonte, MG. Dezembro/76 a Março/77.

\begin{tabular}{|c|c|c|}
\hline \multirow{2}{*}{ Semana } & \multicolumn{2}{|c|}{ Temperatura média $\left({ }^{\circ} \mathrm{C}\right)$} \\
\hline & Ar & Água \\
\hline 1 & 25,0 & 25,0 \\
\hline 2 & 24,5 & 24,7 \\
\hline 3 & 25,7 & 24,0 \\
\hline 4 & 24,7 & 23,5 \\
\hline 5 & 25.0 & 24,0 \\
\hline 6 & 25,0 & 24,5 \\
\hline 7 & 25,3 & 24,5 \\
\hline 8 & 24,3 & 23,6 \\
\hline 9 & 23,6 & 24,0 \\
\hline 10 & 24,0 & 23,5 \\
\hline 11 & 25,8 & 25,2 \\
\hline 12 & 26,8 & 26,5 \\
\hline 13 & 26,0 & 25,0 \\
\hline 14 & 27,5 & 26,2 \\
\hline 15 & 27,5 & 26,7 \\
\hline 16 & 28,2 & 27,7 \\
\hline 17 & 27,1 & 26,5 \\
\hline
\end{tabular}

lizando 110 e $2.41+$ oros respectivamente (Tabela 2).

A distribuiçăo de desivras, segundo intervalus de classe de dez unidades (Tabela 3). mostron que o maior numero delas: $40.8 \%$ (337) continhan 21 a 30 ovos ou $4.0 \%$ (8.516) dos ovos liberados. Neste mesno intervalo de classe, o número médio de ovos/desova foi igual a 25.2 . 
MILWARD-DE-ANDRADE, R. et al. Alimentação e fecundidade de planorbideos criados eni laboratório: IV - Helisoma duryi (Wetherby, 1879), (Pumonata. Plinorbidie). Rer. Salidt publ., S. Paulo, 19:901-8, 1978.

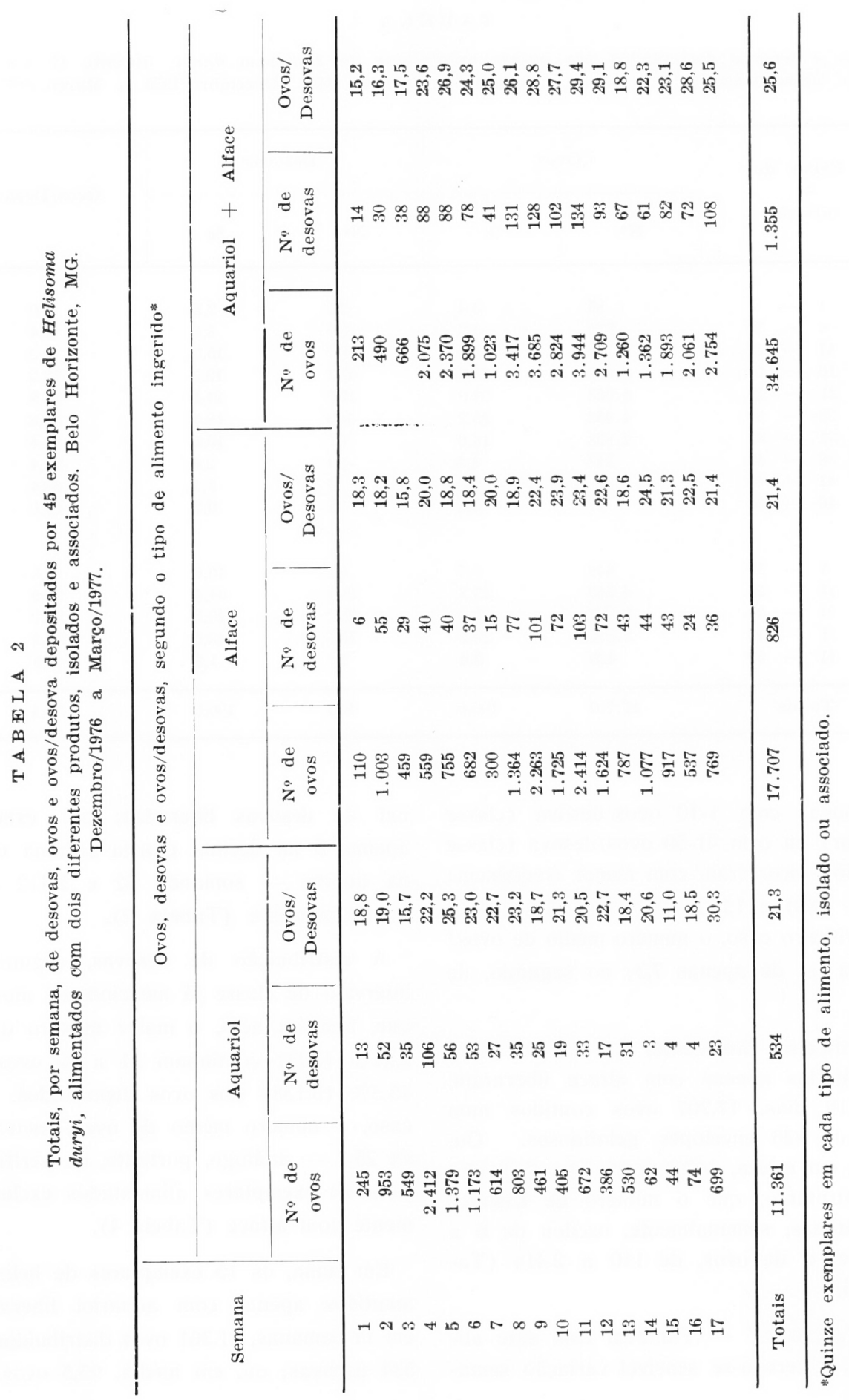


MILWARD-DE-ANDRADE, R. et al. Almentirâa t fecundidade de planorbídeos criados em Jaboratório: IV -. Helisoma dury (Wetherb: 1879). (Pulmonata, Planorbidae). Rer. Saúde

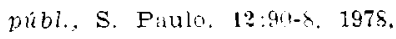

T A B E L A 3

Ovos e desovas depositadas por quinze exemplares de Helisoma duryi, durante 17 semanas e alimentados apenas com alface. Belo Horizonte, MG. Dezembro/1976 a Março/1977.

\begin{tabular}{|c|c|c|c|c|c|c|}
\hline \multirow{2}{*}{\multicolumn{2}{|c|}{$\begin{array}{l}\text { Faixa de } \\
\text { de } \\
\text { variação }\end{array}$}} & \multicolumn{2}{|c|}{ Oros } & \multicolumn{2}{|c|}{ Desovas } & \multirow{2}{*}{ Ovos/Desova } \\
\hline & & No & $\%$ & No & $\%$ & \\
\hline \multicolumn{7}{|l|}{ a) } \\
\hline 1 & -5 & 85 & 0,4 & 21 & 2,5 & 4,0 \\
\hline 6 & -10 & 564 & 3,2 & 67 & 8,3 & 8,4 \\
\hline 11 & -15 & 1.664 & 9,4 & 126 & 15,3 & 9,2 \\
\hline 16 & -20 & 2.879 & 16,3 & 158 & 19,3 & 18,2 \\
\hline 21 & -25 & 4.063 & 23,0 & 177 & 21,5 & 22,9 \\
\hline 26 & -30 & 4.453 & 25,2 & 160 & 18,4 & 27,8 \\
\hline 31 & -35 & 2.823 & 16,0 & 87 & 10,6 & 32,4 \\
\hline 36 & -40 & 747 & 4,2 & 20 & 2,6 & 37,4 \\
\hline 41 & -45 & 382 & 2,1 & 9 & 1,1 & 42,4 \\
\hline 46 & -50 & 47 & 0,2 & 1 & 0,2 & 47,0 \\
\hline \multicolumn{7}{|l|}{ b) } \\
\hline 1 & -10 & 649 & 3,7 & 88 & 10,6 & 7,3 \\
\hline 11 & -20 & 4.543 & 25,7 & 284 & 34,4 & 15,9 \\
\hline 21 & -30 & 8.516 & 48,0 & 337 & 40,8 & 25,2 \\
\hline 31 & -40 & 3.570 & 20,2 & 107 & 13,0 & 33,3 \\
\hline 41 & -50 & 429 & 2,4 & 10 & 1,2 & 42,9 \\
\hline \multicolumn{2}{|c|}{ Totais } & 17.707 & 100,0 & 826 & 100,0 & 21,4 \\
\hline
\end{tabular}

Desovas com 1-10 wos desova (classe inferior) ou com $41-50$ ovos/clesola (classe superior) ocorreram com menor freyuiència: $10.6 \%$ (88) e $1,2 \%$ (10), respectivamente. No primeiro caso, o numero médio de ovos/ desova foi de apenas 7.3 : no segundo, de 42,9 .

Verifica-se, finalmente, yue 15 helisomas alimentados apenas com alface liberaram. cm 119 dias, 17.707 ovos contidos num total de 826 envelopes gelatinosos. Ou. ainda, em média, 148,8 ovos/dia. Salienta$s e$ entretanto, que o número de desovas depositadas, semanalmente, oscilou de $b$ a 103; e, o de ovos, de 110 a 2.414 (Tahela 6$)$.

2. "Ayllariol" - Também com este alimento, observou-se sensivel variação sema- nal de ciesuras liberadas; por exemplo, apenas 3 na décima quarta semana e $100^{\circ}$ na quarta - somando 62 e 2.412 ovos, respectivamente (Tabela 2 ).

A distribuição de desovas, segundo 0 intervalo de classe já mencionado, mostrou que, tambén aчui, o maior numero delas: $38.1 \%$ (203) continham 21 a 30 ovos, ou $45,3 \%$ (5.138) dos ovos depositados. No (aso, o numero médio de ovos/desora foi de 25,3 - análogo, portanto, ao verificado para os exemplares alimentados exclusivamente com alface (Tabela 4 ).

Em suma, os 15 exemplares de helisoma mantidos apenas com aquariol liberaram, em 17 semanas, 11.361 ovos distribuidos em 534 desovas; ou, em média, 95,5 ovos/dia. 
MILWARD-DE-ANDRADE, $R$, et al. Alimentą̧a e fecundidade de planorbídeos criados em laboratório: IV - Helisoma duryi (Wetherby, 1879). (Pulmonata, Planorbidae). Rer. Saúdt públ., S. Paulo, 12:90-8, 1978.

\section{T A B E L A 4}

Ovos e desovas depositados por quinze exemplares de Helisoma duryi, durante 17 semanas e alimentados apenas com "Aquariol". Belo Horizonte, MG. Dezembro/76 a Março/77.

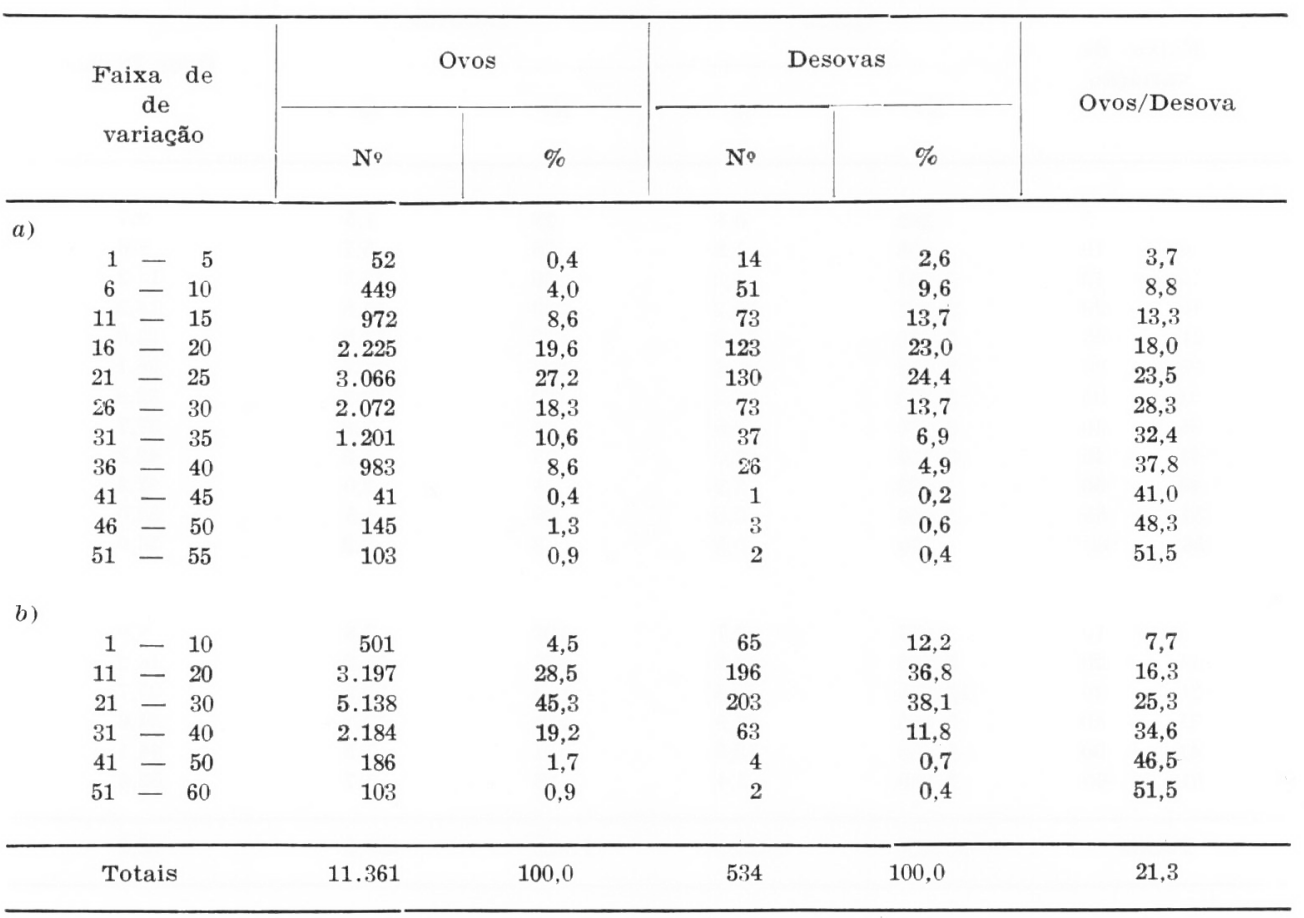

Observa-se, ainda, que os totais de desovas/semana oscilaram de 3 a 106 , somando de 62 a 2.412 ovos, respectivamente. (Tabela 6$)$.

3. Alface + "Aquariol" - Cumu nos casos anteriores, observou-se marcadas variações semanais de desovas liberadas; por exemplo, 14 na primeira semana e 134 na décima primeira - totalizando 213 e 3.944 ovos, respectivamente. Sem embargo, assinala-se, aqui, a ocorrência de número superior a 50 desovas/semana em $76,5 \%$ (13) das 17 semanas consecutivas de observaçōes. Com alface e aquariol, isoladamente, as percentagens alcançadas furam de
$35,3 \%$ (6) e $23,5 \%$ (4), respectivamente (Tabela 2).

Desovas com 1-10 ovos/desovas (classe inferior) ou com 51-60 ovos/desova (classe superior) também ocorreram com menor treqüência: $7.6 \%$ (103) e $1.7 \%$ (23), respectivamente. Nestes casos, os numeros médios de ovos/desova foram, respectivamente, de 8,9 e 52,6 .

o maior número de desovas: $37,3 \%$ (506) continham de 21 a 30 ovos - intervalo de classe no qual foram registrados $37,5 \%$ (13.006) dos ovos depositados pelos 15 exemplares de helisoma observados, enı grupos de cinco em três bacias de plástico. 
MILWARD-DE-ANDRADE, $R$. et il. Alimentaça e fecundidade de planorbídeos criados en laboratório: IV - Helisoma duryi (Wetherby 1879). (Pumonata. Planorbidae). Rev. Saúde publ., S. Paulo. 19:90-5. 1978.

T A B E L A 5

Cvos e desovas depositados por quinze exemplares de Helisoma duryi, durante 17 semanas e alimentados com "Aquariol" + alface fresca. Belo Horizonte, MG. Dezembro/76 a Março/77.

\begin{tabular}{|c|c|c|c|c|c|c|}
\hline \multirow{2}{*}{\multicolumn{2}{|c|}{$\begin{array}{c}\text { Faixa de } \\
\text { variação }\end{array}$}} & \multicolumn{2}{|c|}{ Ovos } & \multicolumn{2}{|c|}{ Desovas } & \multirow{2}{*}{ Ovos/Desova } \\
\hline & & No & $\%$ & $\mathrm{~N} e$ & $\%$ & \\
\hline \multicolumn{7}{|l|}{ a) } \\
\hline 1 & -5 & 262 & 0,8 & 27 & 1,5 & 9,7 \\
\hline 6 & -10 & 655 & 1,9 & 76 & 5,7 & 8,6 \\
\hline 11 & -15 & 1.851 & 5,3 & 140 & 10,3 & 13,2 \\
\hline 16 & -20 & 3.887 & 11,2 & 213 & 15,8 & 18,2 \\
\hline 21 & -25 & 5.521 & 15,9 & 240 & 17,9 & 23,0 \\
\hline 26 & -30 & 7.485 & 21,6 & 266 & 19,7 & 28,1 \\
\hline 31 & $-\quad 35$ & $\mathbf{5 . 6 5 2}$ & 16,3 & 172 & 12,7 & 32,8 \\
\hline 36 & -40 & 3.664 & 10,6 & 97 & 7,2 & 37,7 \\
\hline 41 & -45 & 3.129 & 9,0 & 73 & 5,5 & 42,8 \\
\hline 46 & -50 & 1.329 & 3,9 & 28 & 2,0 & 47,4 \\
\hline 51 & -55 & 1.040 & 3,0 & 20 & 1,5 & 52,0 \\
\hline 56 & -60 & 170 & 0,5 & 3 & 0,2 & 56,0 \\
\hline \multicolumn{7}{|l|}{ b) } \\
\hline 1 & -10 & 917 & 2,7 & 103 & 7,6 & 8,9 \\
\hline 11 & -20 & 5.738 & 16,6 & 353 & 26,0 & 16,2 \\
\hline 21 & -30 & 13.006 & 37,5 & 506 & 37,3 & 25,7 \\
\hline 31 & -40 & 9.316 & 26,9 & 269 & 19,9 & 34,6 \\
\hline 41 & -50 & 4.458 & 12,9 & 101 & 7,5 & 44,1 \\
\hline 51 & -60 & 1.210 & 3,4 & 23 & 1,7 & 52,6 \\
\hline \multicolumn{2}{|c|}{ Totais } & 34.645 & 100,0 & 1.355 & 100,0 & 25,6 \\
\hline
\end{tabular}

T A B E L A 6

Oviposição de quinze exemplares de Helisoma duryi, durante 17 semanas e segundo o tipo de alimento. Belo Horizonte, MG. Dezembro/76 a Março/77).

\begin{tabular}{|c|c|c|c|}
\hline \multirow{2}{*}{ Dados } & \multicolumn{3}{|c|}{ Valores obtidos, segundo o alimento } \\
\hline & Aquariol & Alface & Alface + Aquariol \\
\hline \multicolumn{4}{|l|}{ 1. Desovas: } \\
\hline Totais & 534 & 826 & 1.355 \\
\hline Variação semanal & $3-106$ & $6-103$ & $14-134$ \\
\hline \multicolumn{4}{|l|}{ 2. Ovos: } \\
\hline Totais & 11.361 & 17.707 & 34.645 \\
\hline Variação semanal & $44-2.412$ & $110-2.414$ & $14-3.944$ \\
\hline \multicolumn{4}{|l|}{ 3. Nümero médio de: } \\
\hline Desovas/Dia & 4,5 & 6,9 & 11,4 \\
\hline Desovas/Semana & 31,4 & 48,6 & 79,7 \\
\hline Desovas/Caramujo & 35,6 & 55,1 & 90,3 \\
\hline Ovos/Caramujo & 757,4 & $1.180,5$ & $2.309,7$ \\
\hline Ovos/Dia & 95,5 & 148,8 & 291,1 \\
\hline Ovos/Desova & 21,8 & 21,4 & 25,6 \\
\hline
\end{tabular}


MILWARD-DE-ANDRADE, R. et al, Alimentaça a e fecundidade de planorbídeos criados em laboratório: IV - Helisoma duryi (Wetherby, 1879). (Pulmonata, Planorbidae). Rev. Saúde públ., S. Paulo, 12:90-8, 1978.

Finalmente, ao cabo das 17 semanas ou 119 dias, os 15 exemplares de caramujos criados com ração constituida de aquariol e alface fresca desovaram 1.355 vezes, depositando 34.645 ovos, ou, em média, 291, l ovos/dia. Por semana, o número de desovas variou de apenas 14 até 134 , enyuanto o número de ovos/semana oscilou de também 14 ao elevado total de 3.944 .

Comparativamente, os caramujos alimentados com aquariol e alface associados mostraram notável aumento de fecundidade, como pode ser adiante apreciado.

Alimento Desovas Oros

Aquariol + Alface $1.355(49.9 \%) 34.654(54.4 \%)$ Alface $\quad 826 \quad(30,4 \%) \quad 17.707 \quad(27.8 \%)$ Aquariol $\quad 534(19,7 \%) 11.361 \quad(17,8 \%)$

Totais... $2.715(100,0 \%) 6.3 .713(100.0 \%)$

Singularmente, em contra partida, alface - cujo teor em proteinas é reconhecidamente mediocre, ou seja, cerca de $1,2 \mathrm{~g} /$ $100 \mathrm{~g}$ (Tressler e Jorlyn", 1961) — foi capaz, no caso, de facultar quantidade de ovos nitidamente superior à obtida dos exemplares de helisoma alimentados com aquariol, apenas.

Mortalidade - No decorrer do experimento morreram 42,2\% (19) dos exemplares, sendo, entretanto, substituidos por outros, de igual porte e procedência, na seguinte ordem, por cuba ou bacia plástica utilizada: Cuba-1: um; C-3: cinco; C-4: dois; C-5: três; C-6: cinco; C-7: dois; e, finalmente, C-9: um único exemplar.

\section{DISCUSSÃO}

A introdução, aparentemente recente, de Helisoma (Seminolina) duryi no Brasil, deve despertar interesse que sobrepasse a uma mera e fortuita constatação zoológica - pois, é possivel pensar-se em eventual substituição de hospedeiros intermediários de $S$. mansoni por populações dessa espécie exótica, malgrado o fato de Paraense haver capturado num mesmo biótopo esta e outras espécies de moluscos que poden competir entre si (p. ex., ampularideos $==$ pilídeos e bionfalárias).

O achado de Abdallah e Nasr' deve, por outro lado, ser motivador de novas investigações, particularmente em relação à B. glabrata - como, em verdade, vem fazendo o Laboratório de Ecologia do CPqRR/FIOCRUZ, em Belo Horizonte, MCi.

Os dados apresentados mostram que $H$. duryi pode ser criada facilmente, no laboratório - sendo, ao que parece, espécie mais prolifica que $B$. glabrata.

De fato, como assinalado anteriormente (Milward-de-Andrade e col.", 1974) dez exemplares de $B$. glabrata, alimentados com aquariol e alface conjugados, depositaram 721 desovas e 14.872 ovos, em 13 semanas. Por sua vez, $H$. duryi em numero de quinze espécimens depositaram 1.355 desovas e 34.645 ovos, em 17 semanas.

Em relaçăo a $B$. tenagophila as diferenças parecem ser mais amplas. Pois, num experimento (Milward-de-Andrade e col.2, 1972), dez exemplares depositaram, em 20 semanas, 1.175 desovas e 18.024 ovos, ou seja, quantidades menores que as registradas com helisoma.

No caso de B. straminea, no transcurso de 13 semanas (maio-agosto/1971), foram obtidas 688 desovas e 14.095 ovos provenientes de dez espécimens também alimentados com uma associação de aquariol e alface (Milward-de-Andrade e col. ` 1973).

O sucesso na colonização de um novo habitat, indubitavelmente, depende de numerosas variáveis. $O$ patrimônio genético será, por certo, uma delas, pois, condicionará a capacidade đe adaptação à condições menos favoráveis. Porém, a alimentação será sempre o fator decisivo na criação econômica de caramujos, em condiçōes de laboratório, uma vez que quantidades inadequadas ocasionam reduçăo e, mesmo, suspensão da oviposição. Neste último caso, o mecanismo de defesa face à escassez de nutrientes é a estivação (Milwardde-Andrade e col.s:t 1973). 
MILWARD-DE-ANDRADE, R. et al. Alimentação e fecundidade de planorbídeos criados em laboratório: IV - Helisoma duryi (Wetherby, 1879). (Pulmonata, Planorbidae). Rev. Saúde públ., S. Paulo, 12:90-8, 1978.

RSPUB9/397

MILWARD-DE-ANDRADE, R. et al. IFeeding and fecundity of planorbidae bred in the laboratory: IV - Helisoma duryi (Wetherby, 1879). (Pulmonata, Planorbidae)]. Rev. Saúde públ., S. Paulo, 12:90-8, 1978.

ABSTRACT: After experiments with two types of food, lettuce and "aquariol" (i.e., animal protein flour for ornamental fish) both isolated and in association, in the breeding of Helisoma (Seminolina) duryi (Wetherby, 1879), the following results: were obtained: 1. Snails fed on fresh lettuce yielded, at the end of 17 weeks, 826 egglayings or, better, 17,707 eggs. 2. Specimens fed on "aquariol" produced $534 \mathrm{egg}$ clutches or $11,361 \mathrm{eggs}$. 3. Snails fed on both fresh lettuce and "aquariol" happened to provide 1,355 egglayings or 34,645 eggs. 4. On the average, each batch of 15 snails or three group of 5 ones (each specimen displyaing 10-14 $\mathrm{mm}$ diameter) liberated 95.5, 148.8 and 291.1 eggs/day, in accordance with their type of meal - lettuce, "aquariol" or both foods in association - the number of daily egglayings being, respectively, $4.5,6.9$ and 8.4. 5. The studied specimens were descendent from a strain suposed to be recently introduced in Formosa county, State of Goiás (Brasil) from elsewhere in the Nearctic region (North America). Some remarks on eventual measures for the biological control of Biomphalaria species through the Helisoma duryi, are put forward, since for some time now biological and ecological investigations have been going on.

Uniterms: Planorbidae. Helisoma duryi.

\section{REFERENCIAS BIBLIOGRÁFTCAS}

1. ABDALLAH, A. \& NASR, T. Helisoma duryi as a means of biological control of schistosomiasis vectors snails. $J$. Egypt. med. Ass., 56:514-20, 1973.

2. MILWARD-DE-ANDRADE, R. \& CARVALHO, O. dos S. Alimentação e fecundidade de planorbídeos criados em laboratório: I. Biomphalaria tenagophila (d'Orbigny, 1835). Rev. bras. Biol, 32: $225-33,1972$

3. MILWARD-DE-ANDRADE, R. \& GUIMARÃES, C. T. Sobrevivência de Biomphalaria glabrata (Say, 1818) em jejum: I. Observações com exemplares isolados. Cienc. Cult., 25(Supl. 6):458-9, 1973.

4. MILWARD-DE-ANDRADE, R. \& GUIMAP.ÃES, C. T. Sobrevivência de Biomphalaria glabrata (Say, 1818) em jejum: II. Observações com exemplares agrupados. Cienc. Cult., 25(Supl. 6): $460,1973$.

5. MILWARD-DE-ANDRADE, R. et al, Alimentação e fecundidade de planorbídeos criados em laboratório: II
Biomphalaria straminea (Dunker, 1848). Rev, bras, Biol., 33:119-26, 1973.

6. MILWARD-DE-ANDRADE, R. et al. Alimentação e fecundidade de planorbídeos criados em laboratório: III. Biomphalaria glabrata (Say, 1818). Rev. bras. Malar., 1974. [no prelo]

7. PARAENSE, W. L. Estado atual da sistemática dos planorbídeos brasileiros. Arq. Mus, nac., Rio de Janeiro, 55: 105-28, 1975.

8. PARAENSE, W. L. A natural population of Helisoma duryi in Brazil. Malacologia, 15:369-76, 1976.

9. Tressler, D. I. \& JOSLYN, M. A. Fruit and vegetable juice. Westport, Con., The Avi Publ., 1961.

Recebido nara publicação em 0\%/0\%/197\% Aprovado nara publicação em 14/0r/197r 